\section{Gallbladder cancer: results achieved and future challenges}

\author{
Isidoro Di Carlo*,1 \& Adriana Toro²
}

\section{6th World Congress of International Association of Surgeons Gastroenterologists and Oncologists, Seoul, South Korea, 8-10 September 2016}

This year, the 26th World Congress of the International Association of Surgeons, Gastroenterologists, and Oncologists (IASGO) was hosted by Seoul in South Korea. The congress was extremely well organized, and the quality of the submissions and the relevance of the speakers were excellent. This report highlights the newest and most interesting results regarding the treatment of gallbladder tumors from the conference.

First draft submitted: 28 September 2016; Accepted for publication: 26 October 2016; Published online: 10 November 2016

This year, the 26th World Congress of the International Association of Surgeons, Gastroenterologists and Oncologists (IASGO) was hosted by Seoul in South Korea.

This was the second World Congress of IASGO since its founder, Professor NJ Lygidakis, passed away 2 years ago. But even if the father of this association is no longer with us, his message of promoting the globalization of medical knowledge and maintaining the values of Medicine Beyond Frontiers, remains at the core of the association and its congress.

The Presidents of this year's congress were Ho-Seong Han of Seoul National University, and Si Young Song of Yonsei University of Seoul. The meeting was held under the auspices of the IASGO President, Masatoshi Makuuchi from Japan, and the General Secretaries, Dan G Duda of Harvard University in Boston and Kyoichi Takaori of Kyoto University. The city of Seoul is very well organized and 'runs like clockwork', it is very clean and the people, as per Asiatic tradition, are very kind. The organizing committee of the congress had made considerable efforts and was very welcoming to the meeting attendees.

The congress was extremely well organized, and the quality of the submissions and the relevance of the speakers were excellent. The congress had 1195 participants from 49 countries; the majority of the participants came from Asia. There were four expert consensus meetings, ten special lectures, 37 symposiums, six update lectures, 16 oral presentations sessions, three video festival sessions and three poster sessions. A total of 547 abstracts were accepted for oral or poster presentation; of these, 192 were invited speakers, which included 44 IASGO section leaders.

This report highlights the newest and most interesting results regarding the treatment of gallbladder tumors from the conference.

'Department of Surgical Sciences GF Ingrassia, University of Catania, Cannizzaro Hospital, Catania, Italy

${ }^{2}$ General Surgery, Patti Hospital, Patti (ME), Italy

*Author for correspondence: Tel.: +39 095 726 4863; Fax: +39 095726 3020; idicarlo@unict.it

\section{KEYWORDS}

- cancer • chemotherapy

- gallbladder • polyps

- stones 
Epidemiology \& pathogenesis of gallbladder cancer in different regions of the world

Professor Itaru Endu of Yokohama City University, Japan, focused his presentation on the epidemiology and pathogenesis of gallbladder cancer $(\mathrm{GBC})$ in different regions of the world. GBC incidence varies in different geographical regions; India, South America, eastern Europe and Asia have the highest incidence of GBC. Genomic factors may play a role in this differential distribution of GBC carcinogenesis [1].

The major risk factors for GBC are gallbladder stones, in particular, those that are greater than $3 \mathrm{~cm}$ in diameter or have long-lasting symptoms. In Chile, where GBC is the primary cause of death in women, a strong relationship between gallbladder stones and GBC has been found, especially in Chilean Indian and Hispanic individuals. In these populations, cholesterol lithogenic genes are highly staining [2]. Future work in this field will aim to verify whether lithogenic genomic susceptibility might play a role in GBC carcinogenesis. In Asia, an anomalous pancreaticobiliary junction is associated with GBC, and patients with this anomaly frequently harbor $K R A S$ mutations [3]. Secondary prevention may be possible in these patients through prophylactic laparoscopic cholecystectomies, but further studies are required to validate this approach.

What is the optimal indication for prophylactic cholecystectomy in patients with polyps?

Professor Seung Eun Lee from Chung-Ang University in Seoul, South Korea, focused her presentation on GBC risk associated with gallbladder polyps [4]. Gallbladder polyp diagnoses have increased in recent decades because screening in healthy individuals has increased for various reasons. Accordingly, many people have been diagnosed with gallbladder polyps; the majority of these are benign but a small percentage is associated with an increased risk of cancer development. The aim of Seung Eun Lee's presentation was to determine patients who will benefit from surgery. Currently, patients aged over 50 years with a polyp larger than $10 \mathrm{~mm}$ in diameter are believed to be at an increased risk of cancer development [5]. New studies have indicated a correlation between GBC depth and polyp size. For this reason, patients with gallbladder polyps larger than $10 \mathrm{~mm}$ are recommended to undergo laparoscopic cholecystectomy, even though some of the patients' polyps will be benign.

\section{Increased use of laparoscopy for GBC resection}

Professor Yoo-Seok Yoon from Seoul National University in South Korea reported the current indications for laparoscopic surgery in GBC patients. This procedure has been contraindicated for many years in GBC patients, but with increased proficiency in recent years, this procedure can now be applied; excellent survival results have been reported in initial stage GBC patients. Studies have shown that the 5-year survival rate in T1a and T1b GBC patients that undergo cholecystectomy and lymph node dissection is $100 \%$; the survival in T2 GBC patients, which reaches $90 \%$, is also very encouraging. In T2 GBC patients, GBC laparoscopy has been shown to be associated with distant metastatic recurrence without local recurrence, which is believed to result from a perfect evaluation of the depth of the cancer in the liver. This depth assessment has been achieved through endoscopic and laparoscopic ultrasonography. These two radiological tools can well delineate the layers of the gallbladder. With increased experience, laparoscopy should also allow us to detect liver invasion [6]; better diagnostic definition should extend the use of laparoscopic surgery in GBC patients.

\section{Is chemotherapy or chemoradiation therapy useful for the GBC?}

GBC is associated with a high mortality rate because of late diagnosis and a high rate of recurrence. Surgery is the only cure for GBC patients, but in $\mathrm{T} 3$ and positive-node patients, recurrence is frequent and, consequently, these patients require adjuvant therapy. However, due to the rarity of $\mathrm{GBC}$, no large randomized trials have been performed to determine the best treatment strategy. The following are current recommendations which will need to be further investigated in the future. For patients with a free resection margin, chemotherapy or combined chemoradiotherapy has not been tested in randomized studies. However, many retrospective studies have shown survival advantages associated with both radiation alone and chemoradiotherapy (usually concomitant with fluoropyrimidine); it appears that survival can be improved, but further studies are required [7]. 
A randomized study from Japan [8] investigating treatment with surgery alone or surgery plus two courses of adjuvant chemotherapy (5-fluorouracil and mitomycin) have shown a survival advantage associated with chemotherapy, but the optimal regime needs to be established.

Because data are uncertain, it is recommended that all $>\mathrm{T} 2$ patients who have undergone complete resection, and all patients, excluding T1N0 patients, with a positive margin or positive nodes, are administered chemotherapy (with fluoropyrimidine or gemcitabine) or fluoropyrimidinebased chemoradiotherapy [9]. The European Society of Clinical Oncology suggests using chemoradiotherapy after complete resection [10]. Future studies are required to ascertain how to integrate radiotherapy and chemotherapy, and to establish a uniform protocol.

\section{References}

1 Butte JM, Torres J, Veras EF et al. Regional differences in gallbladder cancer pathogenesis: insights from a comparison of cell cycleregulatory, PI3K, and pro-angiogenic protein expression. Ann. Surg. Oncol. 20, 1470-1481 (2013).

2 Butte JM, Matsuo K, Gönen M et al. Gallbladder cancer: differences in presentation, surgical treatment, and survival in patients treated at centers in three countries. J. Am. Coll. Surg. 212, 50-61 (2011).

3 Morine Y, Shimada M, Takamatsu $\mathrm{H}$ et al. Clinical features of pancreaticobiliary maljunction: update analysis of 2nd Japan-nationwide survey. J. Hepatobiliary Pancreat. Sci. 20, 472-480 (2013).

4 Jang JY, Kim SW, Lee SE et al. Differential diagnostic and staging accuracies of high

\section{Conclusion}

Many aspects regarding GBC are being clarified, especially in respect to prevention. At present, early surgery is the only possible cure for this cancer. Further studies are required to clarify the potential of radiotherapy and/or chemotherapy in GBC.

\section{Financial \& competing interests disclosure}

The authors have no relevant affliations or financial involvement with any organization or entity with a financial interest in or financial conflict with the subject matter or materials discussed in the manuscript. This includes employment, consultancies, honoraria, stock ownership or options, expert testimony, grants or patents received or pending, or royalties.

No writing assistance was utilized in the production of this manuscript. resolution ultrasonography, endoscopic ultrasonography, and multidetector computed tomography for gallbladder polypoid lesions and gallbladder cancer. Ann. Surg. 250, 943-949 (2009).

5 Kwon W, Jang JY, Lee SE, Hwang DW, Kim SW. Clinicopathologic features of polypoid lesions of the gallbladder and risk factors of gallbladder cancer. J. Korean. Med. Sci. 24, 481-487 (2009).

6 Park JH, Kim YH, Kim H et al. Determining the extent of cholecystectomy using intraoperative specimen ultrasonography in patients with suspected early gallbladder cancer. Surg. Endosc. 30, 4229-4238 (2016).

Wang SJ, Lemieux A, Kalpathy-Cramer J et al. Nomogram for predicting the benefit of adjuvant chemoradiotherapy for resected gallbladder cancer. J. Clin. Oncol. 29, 4627-4632 (2011).
8 Takada T, Amano H, Yasuda H et al. Study Group of Surgical Adjuvant Therapy for Carcinomas of the Pancreas and Biliary Tract. Is postoperative adjuvant chemotherapy useful for gallbladder carcinoma? A Phase III multicenter prospective randomized controlled trial in patients with resected pancreaticobiliary carcinoma. Cancer 95 , 1685-1695 (2002).

9 Horgan AM, Amir E, Walter T, Knox JJ. Adjuvant therapy in the treatment of biliary tract cancer: a systematic review and meta-analysis. J. Clin. Oncol. 30, 1934-1940 (2012).

10 Eckel F, Brunner T, Jelic S, ESMO Guidelines Working Group. Biliary cancer: ESMO Clinical Practice Guidelines for diagnosis, treatment and follow-up. Ann. Oncol. 22(Suppl. 6), vi40-vi44 (2011). 